\title{
Grandchildren at High and Low Risk for Depression Differ in EEG Measures of Regional Brain Asymmetry
}

\author{
Gerard E. Bruder, Craig E. Tenke, Virginia Warner, and Myrna M. Weissman
}

Background: Electrophysiologic studies have found abnormalities of alpha asymmetry in depressed adults and offspring of depressed parents, which have been hypothesized to be vulnerability markers of depression. Resting electroencephalogram (EEG) was measured in grandchildren participating in a multigenerational high-risk study.

Methods: Electroencephalogram from 12 electrodes at six homologous sites over each hemisphere (digitally linked-ears reference) was compared in right-handed grandchildren in three groups: 1) both parent and grandparent having major depressive disorder (MDD; $n=19$ ); 2) either parent or grandparent having $\operatorname{MDD}(n=14)$; and 3) neither having $\operatorname{MDD}(n=16)$.

Results: Grandchildren with both depressed parent and grandparent showed greater alpha asymmetry, with relatively less right than left hemisphere activity, when compared with those with neither depressed parent nor grandparent. This difference was present over the parietal region in the eyes-closed condition. Grandchildren having either depressed parent or grandparent also tended to show heightened alpha asymmetry at parietal sites, but they did not differ significantly from those with neither depressed parent nor grandparent. Low-risk grandchildren with neither depressed parent nor grandparent showed no significant alpha asymmetry.

Conclusions: High-risk grandchildren displayed a parietal alpha asymmetry similar to that seen in adolescents or adults having a MDD and in second-generation offspring of parents concordant for MDD. Its presence in high-risk offspring and grandchildren without a lifetime history of MDD supports the hypothesis that an alpha asymmetry indicative of relatively less right than left parietal activity is an endophenotypic marker of vulnerability to a familial form of major depression.

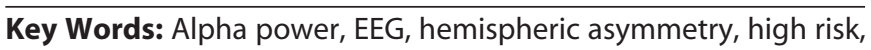
major depressive disorder

$\mathrm{H}$ eightened risk of depressive and anxiety disorders in offspring of parents having a major depressive disorder (MDD) and the early onset of these disorders (Hammen et al. 1990; Warner et al. 1995; Weissman et al. 1987, 1997) underscores the importance of identifying biologic markers of vulnerability for developing a MDD. In the context of a threegeneration, longitudinal high-risk study (Weissman et al. 2005), we have identified two potential electrophysiologic markers of vulnerability: 1) increased startle response in threatening contexts (Grillon et al. 2005) and 2) electroencephalographic (EEG) measures of regional hemispheric activity (Bruder et al. 2005).

The inverse relation of EEG alpha power and cortical activity (Shagass et al. 1972) provides a rationale for using differences in alpha power over right and left hemisphere regions as measures of regional hemispheric asymmetry. Although there have been some conflicting findings, two abnormalities of alpha asymmetry have been reported for depressed subjects. First, an alpha asymmetry indicative of greater right than left frontal activity has been found in depressed adults (Davidson et al. 1987; Gotlib et al. 1998; Henriques and Davidson 1991), women with a history of childhood-onset depression (Miller et al. 2002), and patients having comorbid depressive and anxiety disorders (Bruder et al. 1997). Second, several studies have found the opposite alpha asymmetry at posterior sites, that is, less activity over right than

From the Department of Psychiatry, Columbia University College of Physicians and Surgeons and New York State Psychiatric Institute.

Address correspondence and reprint requests to Gerard Bruder, Ph.D., Department of Biopsychology, Unit 50, New York State Psychiatric Institute, 1051 Riverside Drive, New York, NY10032; E-mail: bruderg@pi.cpmc. columbia.edu.

Received September 7, 2006; revised November 30, 2006; accepted December 6, 2006. left parietal sites, in depressed adolescents or adults (Bruder et al. 1997; Davidson et al. 1987; Kentgen et al. 2000; Reid et al. 1998) and in remitted depressed patients (Henriques and Davidson 1990). On the basis of evidence that offspring of parents concordant for MDD had the highest risk for depressive and anxiety disorders (Nomura et al. 2001), we compared the alpha asymmetry of primarily adult offspring having both parents with MDD, one parent with MDD, or neither parent with MDD (Bruder et al. 2005). Offspring with both parents having a MDD showed greater alpha asymmetry at medial sites than those having one or no parent with MDD, with relatively less activity (greater alpha) over right central and parietal sites. This was evident in high-risk offspring with or without a lifetime diagnosis of MDD, which suggests that heightened alpha asymmetry indicative of relatively less right than left parietotemporal activity may be a marker of vulnerability to MDD.

In this multigenerational longitudinal study (Weissman et al. 2005), grandchildren with both parent and grandparent having a MDD had extremely high rates of psychiatric disorders. In this report, we present for the first time the EEG findings for grandchildren of the original cohort. We examined whether high-risk grandchildren with both depressed parent and grandparent would show an alpha asymmetry similar to that seen for second-generation offspring having both parents with MDD. It was hypothesized that these grandchildren would be at highest risk and would differ from low-risk grandchildren with neither a depressed parent nor grandparent in showing greater alpha asymmetry indicative of relatively less right than left parietotemporal activity.

\section{Methods and Materials}

\section{Subjects}

The probands with MDD, that is, the grandparents, were originally selected from outpatient clinical research settings at Yale University, where they received treatment for mood disorders. The control probands came from a community survey that 
was conducted in New Haven, Connecticut, and had no lifetime history of psychiatric illness based on several direct interviews. The full details of assessments at baseline (Wave 1), 2 years later (Wave 2), and 10 years later (Wave 3) have been previously described (Warner et al. 1999; Weissman et al. 1982, 1992, 1997). A fourth wave of assessments ended 20 years after baseline (Weissman et al. 2005). The lifetime diagnoses are based on extensive interviews concerning medical, psychiatric, social functioning, as well as medical records when available at all four waves over 20 years. Probands, spouses, offspring, and grandchildren were independently interviewed using the Schedule for Affective Disorders and Schizophrenia Lifetime Version (SADS-L) for adults (Mannuzza et al. 1986) and for children between ages 6 and 17, the child version (K-SADS-E; Orvaschel et al. 1982), modified for DSM-IV at Wave 4 (K-SADS-PL; Kaufman et al. 2001), was used. Diagnostic assessments were conducted by mental health professionals with doctoral or master's degrees who received intensive training for interrater reliability and were blind to the clinical status of the parents (Weissman et al. 1997, 2005). Multiple sources of information were obtained, including independent assessments of the offspring by direct interview, parent report, and direct assessment of both biological parents as often as possible. Diagnoses were based on the best estimate procedure (Leckman et al. 1982). A child psychiatrist and child psychologist, who were not involved in the interviewing, independently and blind to the diagnosis of the parent and to prior assessments, reviewed all the material and assigned a DSM-IV diagnosis. Interrater reliability $\kappa$ scores were good to excellent and are described elsewhere (Weissman et al. 1997, 2005). Lifetime diagnoses using DSM-IV criteria at the definite level were cumulative across all four waves over 20 years for grandparents and their offspring, and those for grandchildren were assessed at the fourth wave or for a few at both Waves 3 and 4 . This wave was approved by the institutional review board, and after complete description of the study, written informed consent was obtained from subjects (or parents).

Electrophysiologic tests were obtained during the fourth wave of assessments. The tests included resting EEG followed by startle response measurements that are reported elsewhere (Grillon et al. 2005). To be eligible for these tests, the grandchildren had to be over 7 years old, living in the geographic area of the study, and have no hearing impairment or history of seizures, epilepsy, head trauma, or psychosis. Of the 137 grandchildren who were eligible, 74 participated in the EEG tests. The main reason eligible subjects did not participate is that they declined to be tested. Subjects who did not participate and those who received electrophysiologic tests did not differ significantly in age, gender, or depression status of the probands (Grillon et al. 2005). Seven of these grandchildren did not have usable EEG data, and 18 were not right-handed by self-report. We contrasted the EEG data for the remaining right-handed grandchildren in three groups: 1) the highest risk group having at least one parent and one grandparent with MDD ( $n=19,11$ female subjects); 2$)$ an intermediate risk group having either parent or grandparent with MDD ( $n=14$, 6 female subjects; the alpha asymmetry for grandchildren having either a depressed parent $[n=8]$ or depressed grandparent $[n=6$ ] were highly comparable and did not differ significantly. Their data were therefore combined into one group to avoid small cell sizes.); and 3) the lowest risk group having neither a parent nor grandparent with MDD $(n=16,9$ female subjects). Although these groups did not differ in gender, there were group differences in age at the time of EEG tests ( $F=$ 3.86, $d f=2,46, p<.05)$. Grandchildren with both depressed parent and grandparent $(M=15.4, S D=4.7)$ were older than those with neither depressed parent nor grandparent $(\mathrm{M}=10.6$, $\mathrm{SD}=4.5, p<.05)$, and grandchildren with either parent or grandparent $\mathrm{MDD}$ had an intermediate age $(\mathrm{M}=13.6, \mathrm{SD}=6.2)$ that did not differ significantly from the other groups. Age was not, however, significantly correlated with alpha asymmetry in the total sample $(r=.01, n s)$ or in any of the three groups. Also, when analyses were repeated using age as a covariate, the results remained essentially the same, and all significant group differences in alpha asymmetry were confirmed. Only two grandchildren (both in the highest risk group) were receiving psychoactive medications during the assessments.

\section{Procedures}

Resting EEG was measured while subjects sat quietly during four 2-min periods, with the order of the eyes-open or eyesclosed conditions counterbalanced across subjects (eyes open, closed, closed, open or closed, open, open, closed). Subjects were instructed to remain still and to blink or move their eyes or body as little as possible during the recording periods. In the eyes-open condition, subjects fixated on a cross centered on a computer monitor.

Scalp EEG was measured from 12 electrodes over medial and lateral frontal, central, and parietal regions with half at homologous sites over each hemisphere (F3,F4; F7,F8; C3,C4; T7,T8; P3,P4; P7,P8) using an electrode cap (Electro Cap International, Eaton, Ohio) with linked-ears reference. Tin electrodes were placed at supra- and infraorbital sites surrounding the right eye to monitor eye blinks and vertical eye movements (bipolar), and at right and left outer canthi to monitor horizontal eye movements (bipolar). All electrode impedances were below $5 \mathrm{k} \Omega$. The EEGs were recorded using a Bioamplifier system (James Long Company, New York) at a gain of $10 \mathrm{~K}$ ( $5 \mathrm{~K}$ for eye channels), with a bandpass of .01-30 Hz. A PC-based EEG acquisition system (NeuroScan, Sterling, Virginia) acquired and digitized the data continuously at 100 samples/sec during each recording period.

\section{EEG Analyses}

The EEG data were segmented into consecutive 1.28-sec epochs every $.64 \mathrm{sec}$ ( $50 \%$ overlap). Epochs contaminated by blinks, eye movements, or movement-related artifacts were excluded using a rejection criterion of $\pm 100 \mu \mathrm{v}$ on any channel, followed by interactive rejection of artifacts. The DC offset of each epoch was removed, and the EEG was tapered over the entire 1.28 sec using a Hanning window to suppress spectral side lobes (Bendat and Piersol 1971). The Hanning window deemphasizes data near the beginning and end of each epoch. By overlapping the epochs by $50 \%$, the attenuated data are restored in adjacent epochs, preserving data with minimal redundancy. The EEG data were subjected to an offline power spectrum analysis using a Fast Fourier Transform. Analyses focused on the alpha band, where prior studies have found differences in hemispheric asymmetries for depressed subjects (Bruder et al. 1997; Davidson et al. 1987; Reid et al. 1998). Also, no significant hemispheric asymmetries or interactions involving Group and Hemisphere were found for neighboring frequency bands (theta and low beta). At each electrode, alpha power was averaged for artifact-free epochs spanning each recording period for each condition, and subsequently integrated over 7.0 - to $12.5-\mathrm{Hz}$ band. These alpha band limits were selected after verifying that they adequately sampled the alpha peak for each subject. Logarithms of alpha power were then computed to normalize the data. The total number of minutes of artifact-free EEG data did 
not differ significantly among groups with both depressed parent and grandparent $(M=4.5, S D=1.5)$, either depressed $(M=4.9$, $\mathrm{SD}=1.5)$, and neither depressed $(\mathrm{M}=3.9, \mathrm{SD}=1.5), F=1.66$, $d f=2,46, p=.20$.

\section{Statistical Analyses}

Log power measures at each electrode were submitted to a repeated-measures analysis of variance (ANOVA), using four within-subject factors: Hemisphere (left, right), Anterior-Posterior region (frontal, central, parietal), Medial-Lateral, and Condition (eyes open, eyes closed). Group (neither, either, or both parent/grandparent MDD) and gender (female or male) were used as between-subject factors. When a significant interaction involving Risk Group, Hemisphere, Region, and Condition was found, subsequent analyses followed up this interaction to determine the significance of group differences in hemispheric asymmetry for each condition at frontal, central, and parietal electrode regions. $F$ ratios were evaluated using degrees of freedom computed with the Greenhouse-Geisser $\epsilon$ correction (Jennings and Wood 1976) where appropriate to counteract heterogeneity of variance-covariance matrices with repeated measures.

\section{Results}

Table 1 gives the lifetime diagnoses for grandchildren with neither, either, or both parent and grandparent MDD. Grandchildren with both parent and grandparent MDD had the highest rates of psychopathology, with 57.9\% having at least one psychiatric disorder. They had higher rates of anxiety disorders $\left(\chi^{2}=\right.$ 4.89, $d f=1, p<.05)$ and overall psychopathology $\left(\chi^{2}=3.83\right.$, $d f=1, p<.06$ ) when compared with low-risk grandchildren with neither parent nor grandparent MDD. They also showed higher rates of mood disorders, but these differences did not approach a conventional level of statistical significance. Grandchildren with either parent or grandparent having MDD did not show significantly higher rates. The lifetime rates of psychiatric disorders, as well as the gender and age, for the subset of right-handed grandchildren with sufficient EEG data are comparable to those for the total sample of grandchildren interviewed at the fourth wave (Weissman et al. 2005).
Figure 1 shows the scalp topography of alpha power for grandchildren having neither, either, or both parent/grandparent MDD. Alpha is greatest in the eyes-closed condition at parietal sites (red areas over bottom row of electrodes). There was a marked hemispheric asymmetry of alpha $(F=15.14, d f=1,43$, $p<.001)$, which was greatest in the parietal region (Hemisphere $\times$ Region interaction, $F=14.21, d f=4,86, \epsilon=.73$, $p<.001$ ), and for the eyes-closed condition (Hemisphere $\times$ Region $\times$ Condition interaction, $F=22.32, d f=4,86, \epsilon=.70$, $p<.001)$. Alpha asymmetry in the parietal region with eyes closed was greatest in grandchildren having either or both parent/grandparent with MDD. This was supported by a fourway interaction illustrated in Figure 2 (Group $\times$ Hemisphere $\times$ Region $\times$ Condition, $F=3.20, d f=4,86, \epsilon=.70, p<.05)$. Analyses of simple effects indicated that there was a significant Group $\times$ Hemisphere $\times$ Condition interaction for the parietal region $(F=3.45,2,43, p<.05)$, but not for the frontal or central region. At parietal sites, but not at frontal or central sites, there was a significant alpha asymmetry in the eyes-closed condition, with less activity (greater alpha) over right than left hemisphere, in grandchildren having both parent and grandparent MDD $(F=$ 20.90, $d f=1,43, p<.001$ ) and either parent or grandparent MDD ( $F=12.15, d f=1,43, p<.01)$, but not in those having neither with MDD. In the eyes-closed condition, grandchildren having both depressed parent and grandparent showed greater alpha asymmetry at parietal sites than grandchildren with neither depressed $(F=5.49, d f=1,31, p<.05)$. Grandchildren having either depressed parent or grandparent also tended to have greater alpha asymmetry, but this difference did not achieve a conventional level of significance $(F=3.40, d f=1,26, p=.08)$. There was no significant group difference in alpha asymmetry in the eyes-open condition, and there was no main effect of gender or interaction involving Gender, Group, and Hemisphere, which indicates that the difference in alpha asymmetry among groups was not dependent on gender.

The ANOVA also indicated that there was no significant difference among groups in overall alpha power. Although visual inspection of Figure 1 suggests possible group differences in alpha power in the eyes-closed condition at parietal sites, separate ANOVA of absolute alpha power at parietal sites did not

Table 1. Rates of Lifetime Disorders in Grandchildren

\begin{tabular}{|c|c|c|c|c|c|c|}
\hline & \multicolumn{2}{|c|}{$\begin{array}{c}\text { Neither Parent } \\
\text { nor Grandparent } \\
\text { had MDD } \\
(N=16)\end{array}$} & \multicolumn{2}{|c|}{$\begin{array}{l}\text { Either Parent or } \\
\text { Grandparent had } \\
\text { MDD }(N=14)\end{array}$} & \multicolumn{2}{|c|}{$\begin{array}{l}\text { Both Parent and } \\
\text { Grandparent had } \\
\operatorname{MDD}(N=19)\end{array}$} \\
\hline & $N$ & $\%$ & $N$ & $\%$ & $N$ & $\%$ \\
\hline \multicolumn{7}{|l|}{ Grandchild Diagnoses } \\
\hline Any Anxiety Disorder ${ }^{a}$ & 2 & 12.5 & 3 & 21.4 & 9 & 47.4 \\
\hline Phobia & 2 & 12.5 & 3 & 21.4 & 7 & 36.8 \\
\hline Any Mood Disorder & 1 & 6.2 & 2 & 14.2 & 5 & 26.3 \\
\hline MDD & 1 & 6.2 & 1 & 7.1 & 4 & 21 \\
\hline Dysthymia & 0 & 0 & 1 & 7.1 & 2 & 10.5 \\
\hline Conduct Disorder & 0 & 0 & 1 & 7.1 & 1 & 5.3 \\
\hline Disruptive Disorder & 2 & 12.5 & 0 & 0 & 3 & 15.8 \\
\hline Attention-Deficit Disorder & 0 & 0 & 0 & 0 & 1 & 5.3 \\
\hline Alcohol Abuse & 0 & 0 & 0 & 0 & 1 & 5.3 \\
\hline Drug Abuse & 0 & 0 & 0 & 0 & 3 & 15.8 \\
\hline Any Disorder ${ }^{b}$ & 4 & 25 & 3 & 21.4 & 11 & 57.9 \\
\hline
\end{tabular}

MDD, major depressive disorder.

${ }^{a}$ Difference in rates of anxiety disorders among groups $\left(\chi^{2}=5.66, d f=2, p=.06\right)$.

${ }^{b}$ Difference in rates of any disorder among groups $\left(\chi^{2}=6.02, d f=2, p<.05\right)$. 


\section{MEAN LOG ALPHA POWER (log uv')}

OPEN

\section{CLOSED}
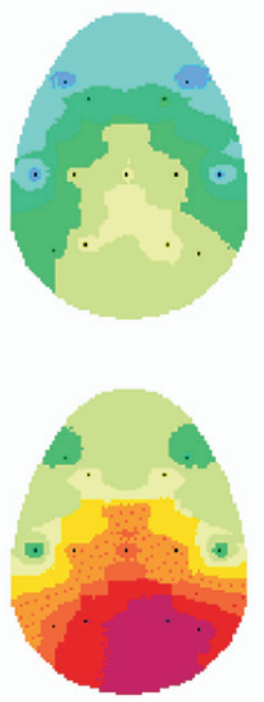

EITHER
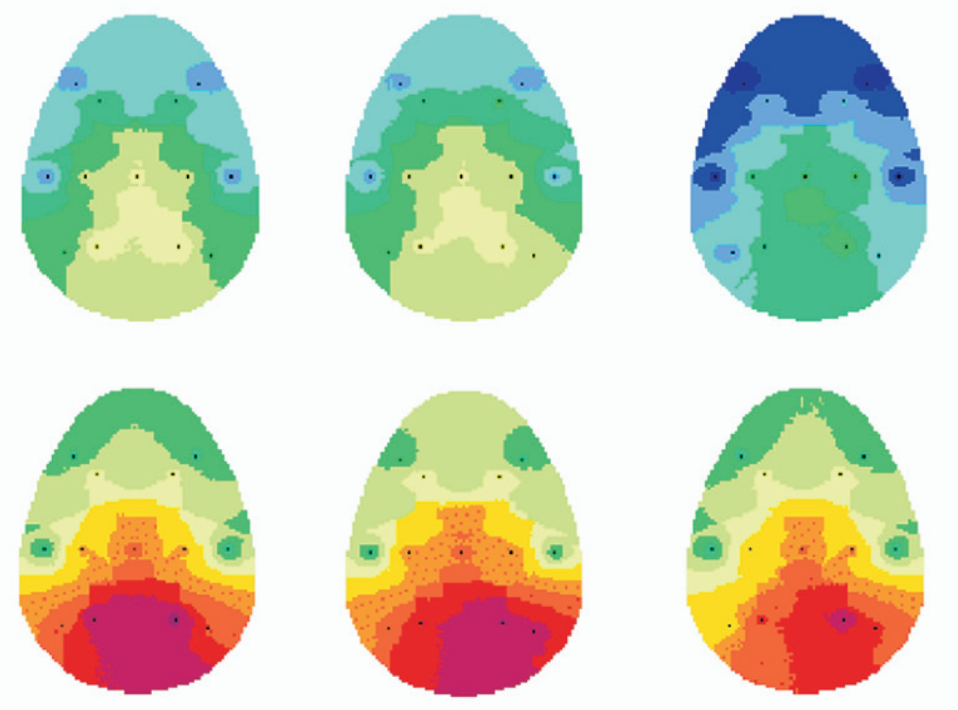

NEITHER PARENT/ GRANDPARENT MDD

\section{B OTH}

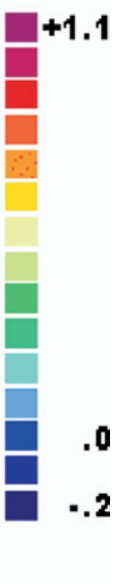

Figure 1. Maps of topography of alpha power for grandchildren with neither, either, or both parent/ grandparent MDD, illustrating that alpha amplitude and asymmetry are greatest in the eyes-closed condition at parietal sites (red areas over bottom row of electrodes). reveal significant group differences over either left $(F=1.18$, $d f=1,46, p=.28)$ or right $(F=.03, d f=1,46, p=.84)$ hemisphere sites.

These analyses included only offspring who were righthanded because handedness could affect asymmetry findings. When the overall ANOVA was repeated including all grandchildren, regardless of handedness, having both ( $n=25 ; 13$ female subjects), either ( $n=22 ; 11$ female subjects), or neither parent/ grandparent MDD ( $n=20 ; 12$ female subjects), the results were the same as for right-handers. Most important, alpha asymmetry at parietal sites with eyes closed was greatest in grandchildren having either or both parent/grandparent MDD, which was supported by a Group $\times$ Hemisphere $\times$ Region $\times$ Condition interaction $(F=3.37, d f=4,122, \epsilon=.80, p<.05)$. There was a significant Group $\times$ Hemisphere $\times$ Condition interaction for the parietal region $(F=3.43, d f=2,61, p<.05)$, but not for the frontal or central regions.

Separate ANOVA were also conducted to compare asymmetries among groups at medial frontal (F4-F3) and lateral frontal (F8-F7) sites. The only significant group difference was found for medial frontal sites $(F=3.76, d f=2,43, p<.05)$ and was similar to that seen at parietal sites (Figure 2), but weaker in magnitude. Grandchildren with either $(\mathrm{M}=.021, \mathrm{SD}=.042)$ or both $(\mathrm{M}=.023, \mathrm{SD}=.038)$ parent/grandparent $\mathrm{MDD}$ had relatively less activity (greater alpha) over the right hemisphere, whereas those with neither parent nor grandparent MDD did not show this frontal asymmetry $(\mathrm{M}=-.008$; $\mathrm{SD}=.039)$.

An important question is whether differences in alpha asymmetry among groups could be due to the greater presence of depressive or anxiety disorders in grandchildren with both parent and grandparent MDD. Few grandchildren had current psychiatric disorders during the fourth wave when the EEG was recorded. Only one grandchild with both parent and grandparent MDD had a current MDD, and none in other groups had a depressive disorder. Three grandchildren had a current anxiety disorder (i.e., simple phobia), two with either parent or grand- parent MDD and one with neither parent nor grandparent MDD. Lifetime diagnoses were therefore used to examine the potential impact of depressive and anxiety disorders on alpha asymmetry differences among groups. The analyses were repeated after excluding grandchildren having a lifetime diagnosis of any mood or anxiety disorder (Table 1). An ANOVA of alpha power for the remaining grandchildren having both ( $n=9 ; 5$ female subjects), either ( $n=11 ; 6$ female subjects), or neither parent/grandparent MDD ( $n=13$; 8 female subjects) yielded essentially the same findings as seen for the total samples. Most important, the difference in alpha asymmetry among groups was significant (Group $\times$ Hemisphere interaction, $F=3.71, d f=2,27, p<.05$ ), and it was again largest over the parietal region in the eyesclosed condition, which was supported by a Group $\times$ Hemisphere $\times$ Region $\times$ Condition interaction $(F=3.34, d f=4,54$, $\epsilon=.65, p<.05$ ). Thus, there was no evidence that the difference in alpha asymmetry among groups was dependent on the presence of depressive or anxiety disorders.

\section{Discussion}

This study examined the effect of depression in parents and grandparents on EEG measures of regional hemispheric activity in grandchildren. Grandchildren with both depressed parent and grandparent, who are at particularly high risk for psychopathology (Weissman et al. 2005), showed greater alpha asymmetry, with relatively less activity over right than left parietal region, compared with low-risk grandchildren with neither depressed parent nor grandparent. Their parietal asymmetry resembles that seen for second-generation offspring of parents concordant for MDD (Bruder et al. 2005) and for adolescents or adults having a depressive disorder (Bruder et al. 1997; Davidson et al. 1987; Kentgen et al. 2000; Reid et al., 1998). Grandchildren having either parent or grandparent MDD tended to show this same alpha asymmetry at parietal sites, but they did not differ significantly from grandchildren with neither depressed parent nor 


\section{EYES CLOSED}
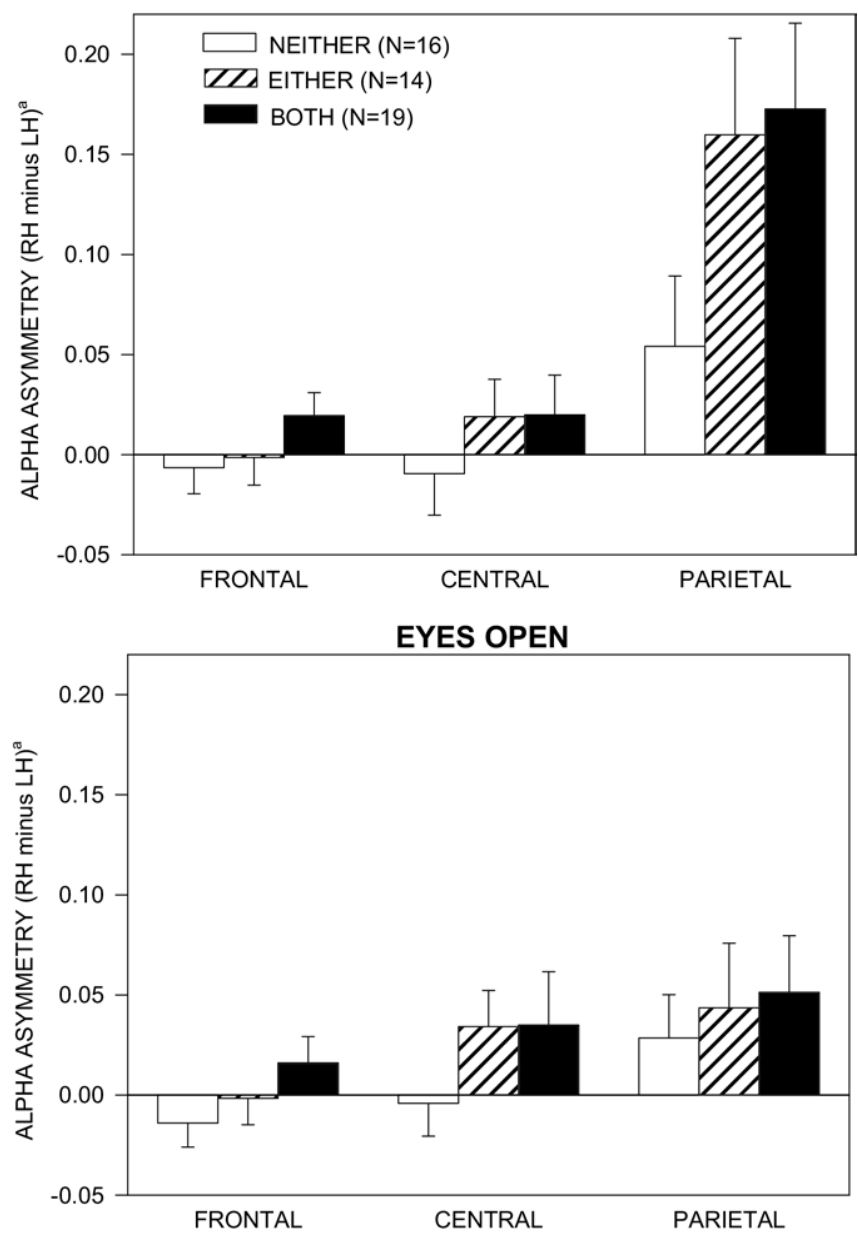

Figure 2. Mean alpha asymmetry with eyes open or closed at frontal, central, and parietal electrode sites for grandchildren with neither, either, or both parent/grandparent MDD (error bars = standard errors). ${ }^{\mathrm{a}} \mathrm{Alpha}$ asymmetry, difference in log alpha power between right hemisphere $(\mathrm{RH})$ and left hemisphere (LH) sites. Positive asymmetry scores indicate greater alpha (less activity) over right than left hemisphere. ${ }^{\text {b } W i t h ~ e y e s ~ c l o s e d, ~ g r a n d c h i l-~}$ dren having both parent/grandparent MDD had significantly greater alpha asymmetry in the parietal region, indicating relatively less right parietal activity, compared with grandchildren having neither parent nor grandparent MDD, $F=5.49, d f=1,31, p<.05$. Those with either parent/grandparent MDD also tended to have greater asymmetry, but this difference did not achieve a conventional level of significance $(F=3.40, d f=1,26, p=.08)$.

grandparent. Although grandchildren with both parent and grandparent MDD had markedly high rates of psychopathology, those with only parent or grandparent MDD did not (Weissman et al. 2005), and it is therefore not surprising that they showed weaker effects. Also, second-generation offspring having only one parent with MDD did not show evidence of heightened parietal asymmetry (Bruder et al. 2005). Low-risk grandchildren with neither depressed parent nor grandparent showed no significant alpha asymmetry, which agrees with our findings for healthy adolescents and adults (Bruder et al. 1997; Kentgen et al. 2000).

The EEG findings for both second and third generations in this longitudinal, high-risk study support the hypothesis that a characteristic asymmetry indicative of relatively less right parietal activity is an endophenotypic marker of vulnerability to a familial form of depression. Findings for EEG and cognitive laterality studies provide additional evidence of relatively less right parietotemporal activity in depression (Bruder 2003; Heller et al. 1995) and indicate that this characteristic asymmetry meets at least some criteria for an endophenotypic marker (Gottesman and Gould 2003; Hasler et al. 2004). The parietal asymmetry is state-independent in that it is found whether or not a depressive disorder is present. Relatively less right than left parietal activity was seen in remitted depressed patients who were normothymic during EEG testing (Henriques and Davidson, 1990). Also, severity of depression, as measured by the Beck Depression Inventory, is not related to parietal alpha asymmetry in depressed patients or adults with a history of depression (Bruder et al. 1997; Vuga et al. 2006). Moreover, depressed patients who responded to an antidepressant showed relatively less right parietal activity both before and after 12 weeks of treatment, which indicates that it is a stable, state-independent characteristic (Sedoruk et al. 2005). Twin studies indicate that EEG power at alpha and other frequencies is one of the most heritable traits in human beings (Smit et al. 2005), and 60\%-70\% of variance of alpha asymmetries are due to individual differences in a temporally stable trait (Hagemann et al. 2005). There is also evidence for specificity of the parietal asymmetry. Although relatively less right parietal activity has been associated with depression, anxious arousal, such as seen in anxiety disorders, is associated with increased right hemisphere activity (Heller et al. 1995), and patients having a comorbid anxiety and depressive disorder showed the opposite parietal asymmetry compared with those having a pure MDD (Bruder et al. 1997). Also, electrophysiologic measures during dichotic listening tests showed that patients having schizophrenia had reduced left as opposed to right parietotemporal activity (Bruder et al. 1999).

The clinical plausibility of this endophenotype is supported by electrophysiologic and dichotic listening findings indicating that hemispheric asymmetries involving parietotemporal regions are related to important clinical features of depression, for example, response to antidepressants and diagnostic subtype. Depressed patients who respond favorably to a selective serotonin reuptake inhibitor (SSRI) show evidence of less right than left hemisphere activity, whereas treatment nonresponders show the opposite asymmetry (Bruder et al. 2001, 2004; Sedoruk et al. 2005). Reduced right hemisphere advantage for perceiving dichotic tones or emotional chimeric faces was found in patients having a MDD with melancholia but not in atypical depression (Bruder et al. 1989, 2002). Although there is evidence for its clinical plausibility, less is known concerning the biological basis of parietal asymmetry. The relation of posterior asymmetry and therapeutic response to an SSRI could suggest a relation to sertonergic function (Bruder, 2003), but its specificity to SSRIs is unknown. Neuroimaging and genetic measures being obtained for family members in this high-risk study should provide new insights into the biological alterations associated with alpha asymmetry and risk for MDD.

On a conceptual level, Davidson (1992) proposed a model in which frontal asymmetry is hypothesized to be a diathesis predisposing individuals to respond with predominately negative or positive affect. Frontal alpha asymmetry in secondgeneration offspring or grandchildren in this study was not, however, associated with MDD in parents or grandparents. Studies in children and adults have identified a number of variables that moderate or mediate the relation of frontal alpha asymmetry and depression or risk for depression (Davidson 1998; Reid et al. 1998). These include temperament (Reid et al. 1998), comorbidity with anxiety (Bruder et al. 1997; Heller and 
Nitschke 1998), mother-infant interactions (Jones et al. 2004), and socioeconomic status (Tomarken et al. 2004), which could contribute to inconsistent findings for frontal alpha asymmetry.

Although frontal asymmetries have been associated with the valence dimension of emotion, Heller et al. (1995) hypothesized that parietotemporal activity plays a key role in the arousal dimension of emotion. In their model, depression involves not only frontal asymmetry but also decreases right parietotemporal activity associated with low emotional arousal. The right parietal region is involved in emotional perception, and there is electrophysiologic evidence that this is abnormal in depressed patients (Deldon et al. 2000; Kayser et al. 2000). Low positive emotionality in children has been associated with both risk for depression and an alpha asymmetry indicative of relatively less right hemisphere activity, particularly over the parietal region (Shankman et al. 2005). There is also evidence that infants of depressed mothers exhibit flat affect and may not develop the skills (i.e., perception and production of emotional expressions) needed for social interactions (Field 1992). Thus, high-risk children with relatively less right parietotemporal activity may be at a disadvantage for perceiving, processing, and responding to positive emotional information, placing them at increased risk for a depression. Although this could account for the relation of alpha asymmetry and risk for depression, alpha asymmetry does not indicate whether the parietal asymmetry in high-risk grandchildren was due to less right or greater left parietal activity. The lack of a group difference in absolute level of alpha over either hemisphere suggests that the difference lies in a relative hemispheric imbalance favoring left over right parietal activity in high-risk grandchildren.

Lifetime rates of anxiety disorders were particularly high in grandchildren with both depressed parent and grandparent. This raises a paradox for the alpha asymmetry findings because anxious arousal is associated with increased right hemisphere activity (Heller et al. 1995), which could counteract the tendency for children at risk for depression to show relatively less right parietotemporal activity. Note, however, that none of the highrisk grandchildren with both depressed parent and grandparent had a current anxiety disorder. Anxiety associated with being in a threatening context (i.e., a testing situation) may, nonetheless, have acted to reduce parietal alpha asymmetry, and yet the trait-related asymmetry in high-risk grandchildren was powerful enough to yield an asymmetry indicating relatively less right parietal activity. Also, it is important to distinguish among various types of anxiety. Anxious apprehension, as opposed to anxious arousal, may actually be associated with increased left hemisphere activity (Heller and Nitschke, 1998). Thus, anxious apprehension or worry in the testing context would not interfere with high-risk grandchildren showing evidence of relatively less right parietotemporal activity.

One limitation of this study is that we did not have ratings of the grandchildren's affect or anxiety during the EEG tests. The extent to which the alpha asymmetry in high-risk grandchildren was related to their emotional state is unclear. As indicated earlier, however, there is evidence that alpha asymmetry in depressed patients is largely state-independent, and heightened parietal asymmetry is present among high-risk grandchildren without lifetime diagnosis of depressive or anxiety disorders. A second limitation is that the EEG measures have low spatial resolution and may not conclusively identify the neuronal generators responsible for an observed asymmetry. This limitation may be addressed by applying generator localization methods
(Tenke et al. 2005) and by supplementing the EEG with neuroimaging measures.

In conclusion, we examined in a multigenerational, high-risk study the EEG patterns in grandchildren of individuals with MDD. An alpha asymmetry indicative of relatively less right than left parietal activity has been observed in second-generation (Bruder et al. 2005) and now third-generation offspring at highest risk, including those without a lifetime history of MDD or anxiety disorders, which supports the hypothesis that it represents an endophenotypic marker of vulnerability to MDD. It is expected that rates of depression and anxiety disorders in the grandchildren will increase as they go through adolescence and adulthood (Kessler et al. 2001; Pine et al. 1998), and the extent to which their alpha asymmetry can predict later onset of MDD will be of particular interest.

This research was supported by the National Institute of Mental Health, Grant NoS. MH36197 (MMW) and MH36295 (GEB). The authors thank Jürgen Kayser for providing us with processing and analysis software. We also thank Jeffrey Hille and Paul Leite for assistance with collection and analysis of the EEG data.

Bendat JS, Piersol AG (1971): Random data: Analyses and measurement procedures. New York: Wiley.

Bruder GE (2003): Frontal and parietotemporal asymmetries in depressive disorders: Behavioral, electrophysiologic, and neuroimaging findings. In: Hugdahl K, Davidson RJ, editors. The asymmetrical brain. Cambridge, MA: MIT Press, 719-742.

Bruder GE, Fong R, Tenke CE, Leite P, Towey JP, Stewart JE, et al. (1997): Regional brain asymmetries in major depression with or without an anxiety disorder: A quantitative electroencephalographic study. Biol Psychiatry 41:939-948.

Bruder GE, Kayser J, Tenke C, Amador X, Friedman M, Sharif Z, Gorman J (1999): Left temporal lobe dysfunction in schizophrenia: Event-related potential and behavioral evidence from phonetic and tonal dichotic listening tasks. Arch Gen Psychiatry 56:267-276.

Bruder GE, Quitkin FM, Stewart JW, Martin C, Voglmaier M, Harrison WM (1989): Cerebral laterality and depression: Differences in perceptual asymmetry among diagnostic subtypes. J Abnorm Psychol 98:177-186.

Bruder GE, Stewart JW, McGrath PJ, Deliyannides D, Quitkin FM (2004): Dichotic listening tests of functional brain asymmetry predict response to fluoxetine in depressed women and men. Neurophsychopharmacology 29:1752-1761.

Bruder GE, Stewart JW, McGrath PJ, Ma GJ, Wexler BE, Quitkin FM (2002): Atypical depression: Enhanced right hemispheric dominance for perceiving emotional chimeric faces. J Abnorm Psychol 3:446-454.

Bruder GE, Stewart JW, Tenke CE, McGrath PJ, Leite P, Bhattacharya N, Quitkin FM (2001): Electroencephalographic and perceptual asymmetry differences between responders and nonresponders to an SSRI antidepressant. Biol Psychiatry 48:416-425.

Bruder GE, Tenke CE, Warner V, Nomura Y, Grillon C, Hille J, et al. (2005): Electroencephalogranphic measures of regional hemispheric activity in offspring at risk for depressive disorders. Biol Psychiatry 57:328-335.

Davidson RJ (1992): Anterior cerebral asymmetry and the nature of emotion. Brain Cog 20:125-151.

Davidson RJ (1998): Anterior electrophysiological asymmetries, emotion, and depression: Conceptual and methodological conundrums. Psychophysiology 35:607-614.

Davidson RJ, Chapman JP, Chapman LJ (1987): Task-dependent EEG asymmetry discriminates between depressed and non-depressed subjects. Psychophysiology 24:585.

Deldon PJ, Keller J, Gergen JA, Miller GA (2000): Right-posterior face processing anomaly in depression. J Abnorm Psychol 109:116-121.

Field T (1992): Infants of depressed mothers. Dev Psychopathol 4:49-66.

Gotlib IH, Ranganath C, Rosenfeld P (1998): Frontal EEG alpha asymmetry, depression, and cognitive functioning. Cog Emotion 12:449-478.

Gottesman II, Gould TD (2003): The endophenotype concept in psychiatry: Etymology and strategic intentions. Am J Psychiatry 160:636-645. 
Grillon C, Warner V, Hille J, Merikangas KR, Bruder GE, Tenke CE, et al. (2005): Families at high and low risk for depression: A three-generation startle study. Biol Psychiatry 57:953-960.

Hagemann D, Hewig J, Seifert J, Naumann E, Bartussek D (2005): The latent state-trait structure of resting EEG asymmetry: Replication and extension. Psychophysiology 42:740-752.

Hammen C, Burge D, Burney E, Adrian C (1990): Longitudinal study of diagnoses in children of women with unipolar and bipolar affective disorder. Arch Gen Psychiatry 47:1112-1117.

Hasler G, Drevets WC, Manji HK, Charney DS (2004): Discovering endophenotypes for major depression. Neuropsychopharmcology 29:1765-1781.

Heller W, Etienne MA, Miller GA (1995): Patterns of perceptual asymmetry in depression and anxiety: Implications for neuropsychological models of emotion and psychopathology. J Abnorm Psychol 104:327-333.

Heller W, Nitschke JB (1998): The puzzle of regional brain activity in depression and anxiety: The importance of subtypes and comorbidity. Cog Emotion 12:421-447.

Henriques JB, Davidson RJ (1991): Left frontal hypoactivation in depression. $J$ Abnorm Psychol 100:535-545.

Henriques JB, Davidson RJ (1990): Regional brain electrical asymmetries discriminate between previously depressed and healthy control subjects. J Abnorm Psychol 99:22-31.

Jennings JR, Wood CC (1976): The epsilon-adjustment procedure for repeated-measures analyses of variance. Psychophysiology 13:277-278.

Jones NA, McFall BA, Diego MA (2004): Patterns of brain electrical activity in infants of depressed mothers who breastfeed and bottle feed: The mediating role of infant temperament. Biol Psychol 67:103124.

Kaufman J, Martin A, King RA, Charney D (2001): Are child-, adolescent-, and adult-onset depression one and the same disorder? Biol Psychiatry 49: 980-1001.

Kayser J, Bruder GE, Tenke CE, Stewart JW, Quitkin FM (2000): Event-related potentials (ERPs) to hemifield presentations of emotional stimuli: Differences between depressed patients and healthy adults in P3 amplitude and asymmetry. Int J Psychophysiology 36:211-236.

Kentgen LM, Tenke CE, Pine DS, Fong R, Klein RG, Bruder GE (2000): Electroencephalographic asymmetries in adolescents with major depression: Influence of comorbidity with anxiety disorders. J Abnorm Psychol 109: 797-802.

Kessler RC, Avenevoli S, Merikangas KR (2001): Mood disorders in children and adolescents: An epidemiologic perspective. Biol Psychiatry 49:10021014.

Leckman JF, Sholomskas D, Thompson D, Belanger A, Weissman MM (1982): Best estimate of lifetime diagnosis: A methodological study. Arch Gen Psychiatry 39:879-883.

Mannuzza S, Fyer AJ, Klein DF, Endicott J (1986): Schedule for Affective Disorders and schizophrenia-lifetime version modified for the study of anxiety disorders (SADS-LA): Rationale and conceptual development. $J$ Psychiatric Res 20:317-325.

Miller A, Fox NA, Cohn JF, Forbes EE, Sherrill JT, Kovacs M (2002): Regional patterns of brain activity in adults with a history of childhood-onset depression: Gender differences and clinical variability. Am J Psychiatry 159:934-940.
Nomura Y, Warner V, Wickramaratne P (2001): Parents concordant for major depressive disorder and the effect of psychopathology in offspring. Psychol Med 31:1211-1222.

Orvaschel H, Puig-Antich J, Chambers W, Tabrizi MA, Johnson R (1982): Retrospective assessment of prepubertal major depression with the Kiddie-SADS-E. J Am Acad Child Adolesc Psychiatry 21:392-397.

Pine D, Cohen P, Gurley D, Brook J, Ma Y (1998): The risk for early-adulthood anxiety and depressive disorders in adolescents with anxiety and depressive disorders. Arch Gen Psychiatry 55:56-64.

Reid SA, Duke LM, Allen JJB (1998): Resting frontal electroencephalographic asymmetry in depression: Inconsistencies suggest the need to identify mediating factors. Psychophysiology 35:389-404.

Sedoruk JP, Tenke CE, Stewart JW, McGrath PJ, Bruder GE (2005): EEG alpha predictors of fluoxetine response are unchanged by treatment. Psychophysiology 42:S69.

Shagass C (1972): Electrical activity of the brain. In: Greenfield NS, Sternbach RA, editors. Handbook of Psychophysiology. New York: Holt, Rinehart \& Winston, 263-328.

Shankman SA, Tenke CE, Bruder GE, Durbin CE, Hayden EP, Klein DN (2005): Low positive emotionality in young children: Association with EEG asymmetry. Dev Psychopathology 17:85-98.

Smit DJA, Posthuma D, Boomsma DI, De Geus EJC (2005): Heritability of background EEG across the power spectrum. Psychophysiology 42:691697.

Tenke CE, Kayser J (2005): Reference-free quantification of EEG spectra: Combining current source density (CSD) and frequency principal components analysis (fPCA). Clin Neurophysiol 116:2826-2846.

Tomarken AJ, Dichter GS, Garber J, Simien C (2004): Resting frontal brain activity: Linkages to maternal depression and socio-economic status among adolescents. Biol Psychol 67:77-102.

Vuga M, Fox NA, Cohn JF, George CJ, Levenstein RM, Kovacs M (2006): Long-term stability of frontal electroencephalographic asymmetry in adults with a history of depression and controls. Int J Psychophysiology 59:107-115.

Warner V, Mufson L, Weissman MM (1995): Offspring at high and low risk for depression and anxiety: Mechanisms of psychiatric disorder. J Am Acad Child Adolesc Psychiatry 34:786-797.

Warner V, Weissman MM, Mufson L, Wickramaratne PJ (1999): Grandparents, parents, and grandchildren at high risk for depression: A three-generation study. J Am Acad Child Adolesc Psychiatry 38:289-296.

Weissman MM, Fendrich M, Warner V, Wickramaratne P (1992): Incidence of psychiatric disorder in offspring at high and low risk for depression. J Am Acad Child Adolesc Psychiatry 31:640-648.

Weissman MM, Gammon GD, John K, Merikangas KR, Warner V, Prusoff BA, et al. (1987): Children of depressed parents: Increased psychopathology and early onset of major depression. Arch Gen Psychiatry 44:847-853.

Weissman MM, Kidd KK, Prusoff BA (1982): Variability in rates of affective disorders in relatives of depressed and normal probands. Arch Gen Psychiatry 39:1397-1403.

Weissman MM, Warner V, Nomura Y, Verdeli H, Pilowsky D, Bruder G, et al. (2005): Offspring at high and low risk for depression: A three generation study. Arch Gen Psychiatry 62:29-36.

Weissman MM, Warner V, Wickramaratne P, Moreau D, Olfson M (1997): Offspring of depressed parents: Ten years later. Arch Gen Psychiatry 54: 932-940. 